\title{
JOIN-PRINCIPAL ELEMENTS IN NOETHER LATTICES
}

\author{
E. W. JOHNSON AND J. P. LEDIAEV
}

\begin{abstract}
In this paper we determine the structure of joinprincipal elements in Noether lattices and we apply these results to obtain the Krull Principal Ideal Theorem for join-principal elements, a representation theorem for a class of Noether lattices, and some interesting ring results.
\end{abstract}

The concept of a Noether lattice, introduced by R. P. Dilworth [1], is an abstraction of the lattice of ideals $L$ of a Noetherian ring $R$. Fundamental to this abstraction is the following lattice-theoretic characterization of two key properties of a principal ideal $E:(A \wedge B: E) E=A E \wedge B$ and $(A \vee B E): E=(A: E) \vee B$ for all $A, B \in L$. An element $E$ of a multiplicative lattice $\mathscr{L}$ is said to be a meet-principal (join-principal) element if it satisfies the first (second) identity. $E$ is principal if it satisfies both identities.

The concepts of meet- and join-principalness have been instrumental in the generalization of classical results of the ideal theory of Noetherian rings to lattices ([1], [2], and [4]), and have provided a basis for representation and embedding theorems for certain classes of Noether lattices ([3], [4], and [5]). The results of [3], [4], and [5] hint that, in general, the connection between meet- and join-principal elements in a Noether lattice may be quite close. In this paper, we obtain a structural result for join-principal elements (Theorem 1) which not only allows us to explore the relationship between meet- and join-principal elements but it also yields as corollaries the Krull Principal Ideal Theorem for joinprincipal elements, the representation theorem in [4], the result that joinprincipal maximal elements have minimal bases consisting of independent elements, and some interesting ring theoretic results.

We adopt the terminology of [1].

Let $\mathscr{L}$ be a noether lattice and let $M$ and $E$ be elements of $\mathscr{L}$ such that $E$ is meet-principal and $M$ is maximal. Then $\{E\}$ is meet-principal, and therefore join-irreducible, in $\mathscr{L}_{. Y}$. It follows that $\{E\}$ is principal in $\mathscr{L}_{. I}$,

Received by the editors June 1, 1971.

AMS 1970 subject classifications. Primary 06A20, 13A15; Secondary 13F05.

Key nords and phrases. Noether lattice, join-principal element, meet-principal element, principal element, Krull Principal Ideal Theorem, Noetherian ring, general ZPI-ring, Dedekind domain, weak union condition.

(c) American Mathematical Society 1972 
and hence that $((B \vee C E): E)_{M}=((B: E) \vee C)_{M}$, for all $B, C \in \mathscr{L}$. Since this is true for every maximal element $M$, this establishes the following: If $E$ is a meet-principal element of a Noether lattice $\mathscr{L}$, then $E$ is joinprincipal. The converse of this result is, however, not valid in general. To see this, let $\left(\mathscr{L}_{i}, M_{i}\right)$ be local Noether lattices $(1 \leqq i \leqq n)$, and let $\mathscr{L}$ be the sub-multiplicative-lattice of $\mathscr{L}_{1} \oplus \cdots \oplus \mathscr{L}_{n}$ defined by $\mathscr{L}=$ $\left(M_{1} / O \oplus \cdots \oplus M_{n} / O\right) \cup\{(I, \cdots, I)\}$. Then $\mathscr{L}$ is a Noether lattice in which an element $A=\left(A_{1}, \cdots, A_{n}\right)(A \neq I)$ is join-principal if and only if each of the coordinates $A_{i}$ is join-principal $(i=1, \cdots, n)$. On the other hand, $A$ is meet-principal if and only if each of the coordinates $A_{i}$ is meetprincipal and at most one of the $A_{i}$ is different from 0 . Hence, this method of construction defines a large class of Noether lattices which have many join-principal elements that are not meet-principal.

We note that, in the above example, any join-principal element $A=$ $\left(A_{1}, \cdots, A_{n}\right)$ is decomposable into coordinates $\left(0, \cdots, A_{i}, \cdots, 0\right)$ any two of which annihilate each other. Theorem 1 shows that this is a basic structural property of join-principal elements.

THEOREM 1. Let $\mathscr{L}$ be a local Noether lattice and let $A$ be a joinprincipal element of $\mathscr{L}$. Then $A$ has a minimal base $E_{1}, \cdots, E_{k}$ such that $E_{i} E_{j}=0$ whenever $i \neq j$

Proof. Let $F_{1}, \cdots, F_{r}(r \geqq 0)$ be a minimal collection of principal elements such that $F_{i} \leqq A \wedge(0: A), \quad i=1, \cdots, r$, and $\bigvee_{i=1}^{r}\left(F_{i} \vee M A\right)=$ $(A \wedge(0: A)) \vee M A$. Extend the collection $F_{1}, \cdots, F_{r}$ to a minimal base $F_{1}, \cdots, F_{r}, \cdots, F_{k}$ for $A$, and for each $i=1, \cdots, k$, set $C_{i}=F_{1} \vee \cdots \vee$ $\hat{F}_{i} \vee \cdots \vee F_{k}$. Since $A$ is join-principal and $A^{2}=A C_{i} \vee F_{i}^{2}$,

$$
\begin{aligned}
A & =\left(A^{2}: A\right) \wedge A=\left(\left(A C_{i} \vee F_{i}^{2}\right): A\right) \wedge A \\
& =\left(C_{i} \vee\left(F_{i}^{2}: A\right)\right) \wedge A=C_{i} \vee\left(\left(F_{i}^{2}: A\right) \wedge A\right) .
\end{aligned}
$$

Also,

Hence

$$
\begin{aligned}
F_{i} \vee\left(\left(F_{i}^{2}: A\right) \wedge A\right) & =\left(\left(A F_{i} \vee F_{i}^{2}\right): A\right) \wedge A=\left(A F_{i}: A\right) \wedge A \\
& =\left(F_{i} \vee(0: A)\right) \wedge A=F_{i} \vee((0: A) \wedge A)
\end{aligned}
$$

$$
\begin{aligned}
\left(F_{i}^{2}: A\right) \wedge A & =\left(\left(F_{i}^{2}: A\right) \wedge A\right) \wedge\left(F_{i} \vee((0: A) \wedge A)\right) \\
& =((0: A) \wedge A) \vee\left(F_{i}^{2}: A\right) \wedge F_{i}=((0: A) \wedge A) \vee\left(F_{i}^{2}: A F_{i}\right) F_{i},
\end{aligned}
$$

and therefore,

$$
A=C_{i} \vee((0: A) \wedge A) \vee\left(F_{i}^{2}: A F_{i}\right) F_{i} .
$$

Assume $s>r$. Then $(0: A) \wedge A \leqq C_{s} \vee M A$, so by the Intersection Theorem, $A=C_{s} \vee\left(F_{s}^{2}: F_{s} A\right) F_{s}$. Since $A \neq C_{s}$, it follows that $F_{s}^{2}: F_{s} A=I$, and hence 
that $F_{s} A=F_{s}^{2}$. On the other hand, if $s \leqq r$, then $F_{s} \leqq 0: A$, and so $F_{s} A=$ $F_{s}^{2}=0$. Therefore $F_{i} A=F_{i}^{2}, i=1, \cdots, k$. Hence

$$
\begin{aligned}
A & =\left(A F_{i}: F_{i}\right) \wedge A=\left(F_{i}^{2}: F_{i}\right) \wedge A \\
& =\left(F_{i} \vee\left(0: F_{i}\right)\right) \wedge A=F_{i} \vee\left(\left(0: F_{i}\right) \wedge A\right), \quad i=1, \cdots, k .
\end{aligned}
$$

Now, assume that $s$ is a nonnegative integer such that $r \leqq s \leqq k-1$ and $\left(F_{1} \vee \cdots \vee F_{i}\right)\left(F_{i+1} \vee \cdots \vee F_{k}\right)=0$ for all $i=r, \cdots, s$ (if $i=0$, then $\left.F_{1} \vee \cdots \vee F_{i}=0\right)$. Then $A=F_{s+1} \vee\left(\left(0: F_{s+1}\right) \wedge A\right)$, so $F_{s+1} \vee \cdots \vee F_{k}=F_{s+1} \vee$ $\left(\left(F_{s+1} \vee \cdots \vee F_{k}\right) \wedge\left(0: F_{s+1}\right)\right) \quad$ and $\quad A=F_{1} \vee \cdots \vee F_{s+1} \vee\left(\left(F_{s+1} \vee \cdots \vee F_{k}\right) \wedge\right.$ $\left.\left(0: F_{s+1}\right)\right)$. For $1 \leqq i \leqq s+1$, set $F_{i}^{\prime}=F_{i}$. Then $F_{1}^{\prime}, \cdots, F_{s+1}^{\prime}$ can be extended to a minimal base $F_{1}^{\prime}, \cdots, F_{k}^{\prime}$ for $A$ using principal elements

$$
F_{s+2}^{\prime}, \cdots, F_{k}^{\prime} \leqq\left(F_{s+1} \vee \cdots \vee F_{k}\right) \wedge\left(0: F_{s+1}\right) \text {. }
$$

Hence $\left(F_{1}^{\prime} \vee \cdots \vee F_{i}^{\prime}\right)\left(F_{i+1}^{\prime} \vee \cdots \vee F_{k}^{\prime}\right)=0$ for each $i=r, \cdots, s+1$. By induction, it now follows that $A$ has a minimal base $E_{1}, \cdots, E_{k}$ such that $\left(E_{1} \vee \cdots \vee E_{i}\right)\left(E_{i+1} \vee \cdots \vee E_{k}\right)=0$ for all $i=0, \cdots, k-1$. Q.E.D.

We now explore some of the consequences of Theorem 1. We begin with an extension of a result of [5] which was instrumental in determining the embeddability of distributive local Noether lattices with join-principal maximal elements.

Corollary 1. Let $(\mathscr{L}, M)$ be a local Noether lattice in which $M$ is join-principal. Then $M$ has a minimal base $E_{1}, \cdots, E_{k}$ of independent elements.

Proof. Let $E_{1}, \cdots, E_{k}$ be a minimal base for $M$ such that $E_{i} E_{j}=0$ whenever $i \neq j$. We begin by showing that $E_{i} \wedge E_{j}=0$ for $i \neq j$. Hence, let $E$ be a principal element such that $E \leqq E_{i} \wedge E_{j}$. Assume $E \neq 0$. Choose $r$ such that $E \leqq E_{i}^{r}$ and $E E_{i}^{r+1}$. And choose $s$ so that $E \leqq E_{j}^{s}$ and $E E_{j}^{s+1}$. Then $E \leqq E_{i}^{r}$ and $E M E_{i}^{r}=E_{i}^{r+1}$, so $E=E_{i}^{r}$. Similarly, $E=E_{j}^{s}$. But then $M E_{i}^{r-1}=M E_{j}^{s-1}$, so $E_{i}^{r-1} \vee(0: M)=E_{j}^{s-1} \vee(0: M)$, and

$$
E=E_{i}^{r}=E_{i}\left(E_{i}^{r-1} \vee(0: M)\right)=E_{i}\left(E_{j}^{s-1} \vee(0: M)\right)=0 .
$$

Hence $E_{i} \wedge E_{j}=0$. Since $M$ is join-principal in $\mathscr{L} \mid E_{k}$, the result follows by induction on $k$.

COROLlARY 2. Let $\mathscr{L}$ be a Noether lattice in which, for every maximal element $M, O_{M}$ is meet-irreducible. If every maximal element is joinprincipal, then every element of $\mathscr{L}$ is principal and $\mathscr{L}$ is representable as a lattice of ideals of a Noetherian ring.

Proof. Since, for every maximal element $M,\{O\}$ is meet-irreducible and $\{M\}$ is join-principal in $\mathscr{L}_{M}$, it follows that $\{M\}$ is principal in $\mathscr{L}_{M}$. 
Hence, by the results of [3], every element of $\mathscr{L}$ is principal and $\mathscr{L}$ is representable. Q.E.D.

From Corollary 2 and the results of [3], we deduce the following new characterization of general ZPI-rings (i.e., Noetherian multiplication rings): A Noetherian ring $R$ is a general ZPI-ring if and only if for each maximal ideal $M$ of $R, M$ is join-principal in the lattice of ideals of $R$ and $O_{M}$ is meet-irreducible.

In [4] we showed that if a maximal element $M$ is join-principal and 0 is prime, then $M$ is principal. The following Corollary 3 extends this result to an arbitrary join-principal element. Also, Corollary 3 and the results of [3] yield the following new characterization of Dedekind domains: A Noetherian domain $D$ is Dedekind if and only if every maximal ideal of $D$ is a join-principal element in the lattice of ideals of $D$.

Corollary 3. Let $\mathscr{L}$ be a Noether lattice. Let $E$ be an element of $\mathscr{L}$ which is either meet-or join-principal, and let $P$ be a prime element in $\mathscr{L}$. Then $E \vee P$ is principal in $\mathscr{L} \mid P$. In particular, if 0 is prime in $\mathscr{L}$, then every join-principal element is principal.

Corollary 3 provides an alternative proof for our extension of the Krull Principal Ideal Theorem to join-principal elements [4]. We state this result as Corollary 4 and we note that since there is a large class of Noether lattices which have many join-principal elements that are not principal, Corollary 4 represents a strengthening of the Principal Ideal Theorem in Noether lattices [1].

COROllaRy 4. Let $\mathscr{L}$ be a Noether lattice and let $E$ be an element of $\mathscr{L}$ which is either meet-or join-principal. If $P$ is a minimal prime of $E$, then $P$ has rank at most 1 .

Proof. The proof is clear, by Corollary 3 and the Principal Ideal Theorem for principal elements [1].

A Noether lattice $\mathscr{L}$ satisfies the weak union condition if, given elements $A, B$, and $C$ such that $A \leq B$ and $A \leq C$, it follows that there exists a principal element $E \leqq A$ such that $E \leq B$ and $E \leq C$. This concept was used in [3] to characterize the distributive Noether lattices which are representable. A lattice of ideals of a commutative ring with an identity clearly satisfies this condition.

Let $\mathscr{L}$ be a local Noether lattice. Then $\mathscr{L}$ satisfies the weak union condition if and only if given any principal elements $A_{1}, \cdots, A_{k}$ in $\mathscr{L}$, there exists a principal element $A$ such that $A \vee\left(\bigvee_{i \neq j} A_{i}\right)=\bigvee_{i=1}^{k} A_{i}$ for all $j=1, \cdots, k$. To see this, assume that $\mathscr{L}$ satisfies the weak union condition, and let $A_{1}$ and $A_{2}$ be principal elements such that $A_{1} A_{2}$ and $A_{2} \leq A_{1}$. 
By the Intersection Theorem, $A_{1} A_{2} \vee M A_{1}$ and $A_{2} A_{1} \vee M A_{2}$, so there exists a principal element $A \leqq A_{1} \vee A_{2}$ such that $A A_{2} \vee M A_{1}$ and $A+A_{1} \vee$ $M A_{2}$. Then

$$
\begin{aligned}
\left(A_{1} \vee A_{2}\right) /\left(A_{1} \vee M A_{2}\right) & \cong A_{2} /\left(A_{1} \vee M A_{2}\right) \wedge A_{2} \\
& =A_{2} /\left[\left(A_{1} \vee M A_{2}\right): A_{2}\right] A_{2}=A_{2} / M A_{2},
\end{aligned}
$$

and so $\left(A_{1} \vee A_{2}\right) /\left(A_{1} \vee M A_{2}\right)$ is simple. Hence, $A \vee A_{1} \vee M A_{2}=A_{1} \vee A_{2}$ and similarly $A \vee A_{2} \vee M A_{1}=A_{1} \vee A_{2}$. Therefore, $A \vee A_{1}=A_{1} \vee A_{2}=A \vee A_{2}$, by the Intersection Theorem. The general result follows by induction on $k$. The converse is clear.

In view of the above statement, it is now easy to see that if $\mathscr{L}$ is a local Noether lattice which satisfies the weak union condition, then, given primes $P_{1}, \cdots, P_{k}$ and an element $A$ such that $A \pm P_{i}(i=1, \cdots, k)$, there exists a principal element $E \leqq A$ such that $E \leqq P_{i}(i=1, \cdots, k)$.

The above observations lead to a straightforward generalization of the ring theoretic proof $[6$, p. 406$]$ of the following lemma.

Lemma. Let $\mathscr{L}$ be a local Noether lattice which satisfies the weak union condition. And let $A, B \in \mathscr{L}$ such that $A: B=A$. Then there exists a principal element $E \leqq B$ such that $E \leq M^{2}$ and $A: E=A$.

COROLlaRY 5. Let $\mathscr{L}$ be a Noether lattice which satisfies the weak union condition, and let $E$ be a join-principal element of $\mathscr{L}$. Then either $E$ is a zero divisor (i.e., $E A=0$ for some $A \neq 0 \in \mathscr{L}$ ) or $E$ is a principal element.

Proof. Assume that $E$ is not a zero divisor in $\mathscr{L}$, and let $M$ be any maximal element such that $E \leqq M$. Then $E \leqq P$ for any prime $P$ of 0 in $\mathscr{L}$, and so $0:\{E\}=0$ in $\mathscr{L}_{M}$. Since $\mathscr{L}_{{ }_{M}}$ inherits the weak union condition, by the lemma there is a principal element $\left\{E_{1}\right\} \leqq\{E\}$ such that $0:\left\{E_{1}\right\}=0$ and $\left\{E_{1}\right\}\{M\}\{E\}$. Using the technique of the proof of Theorem 1, we can show that $\{E\}$ has a minimal base $\left\{E_{1}\right\}, \cdots,\left\{E_{k}\right\}$ where $\left\{E_{i}\right\}\left\{E_{j}\right\}=0$ for $i \neq j$. But $0:\left\{E_{1}\right\}=0$ implies that $k=1$, so $\{E\}=\left\{E_{1}\right\}$. Since $\{E\}$ is principal in $\mathscr{L}_{\lambda I}$ for every maximal element $M$ in $\mathscr{L}$, we conclude that $E$ is principal in $\mathscr{L}$. Q.E.D.

Using the construction process given earlier in the paper, we can easily see that a Noether lattice which does not satisfy the weak union condition may have many join-principal elements which are neither zero divisors nor principal. If $\left(\mathscr{L}_{i}, M_{i}\right)(1 \leqq i \leqq n$ and $n \geqq 2)$ are regular local Noether lattices of altitude 1 , then any $\left(A_{1}, \cdots, A_{n}\right) \in M_{1} / O \oplus \cdots \oplus M_{n} / O$, $A_{i} \neq 0$ for each $i$, is an example of such an element.

Since a meet-principal element of a lattice of ideals $L$ of a local Noetherian ring $R$ is join-irreducible in $L$, it is a principal ideal in $R$ [2]. 
In general, this is not true for join-principal elements of $L$. However, since $L$ satisfies the weak union condition and principal elements in $L$ are principal ideals, we have the following corollary.

COROllary 6. Let $L$ be the lattice of ideals of a local Noetherian ring $R$, and let $E$ be a join-principal element of $L$. Then $E$ is a zero divisor in $L$ or $E$ is a principal ideal of $R$.

\section{REFERENCES}

1. R. P. Dilworth, Abstract commutative ideal theory, Pacific J. Math. 12 (1962), 481-498. MR 26 \#1333.

2. E. W. Johnson, A-transforms and Hilbert functions in local lattices, Trans. Amer. Math. Soc. 137 (1969), 125-139. MR 38 \#5675.

3. E. W. Johnson and J. P. Lediaev, Representable distributive Noether lattices, Pacific J. Math. 28 (1969), 561-564. MR 41 \#118.

4. - Join-principal elements and the principal-ideal theorem, Michigan Math. J. 17 (1970), 255-256. MR 41 \#8311.

5. - Structure of Noether lattices with join-principal maximal elements, Pacific J. Math. 37 (1971), 101-108.

6. O. Zariski and P. Samuel, Commutative algebra. Vol. 2, University Series in Higher Math., Van Nostrand, Princeton, N.J., 1960. MR 22 \#11006.

Department of Mathematics, University of Iowa, Iowa City, Iowa 52240 